International Journal of Computer Networks \& Communications (IJCNC) Vol.3, No.4, July 2011

\title{
INTELLIGENT VERTICAL HANDOVER FOR HETEROGONOUS NETWORKS USING FL AND ELECTRE
}

\author{
Mohammed M. Alkhawlani \\ Faculty of Science and Engineering, University of Science and Technology, Sana'a, \\ Yemen \\ m.alshadadidust.edu.ye
}

\begin{abstract}
The future Heterogeneous Wireless Network (HWN) is composed of multiple Radio Access Technologies (RATs) and domains, therefore, new Radio Resource Management (RRM) schemes and mechanisms are necessary to benefit from the individual characteristics of each RAT and to exploit the gain resulting from jointly considering the whole set of the available radio resources in each RAT. Vertical Handover (VHO) enables users to access several networks such as WLAN, WMAN, WPAN, and WWAN in parallel. It allows the applications even the real time application to be seamlessly transferred among different networks.

In this paper, a decision support system is developed to address the VHO problem. This system combines fuzzy logic and ELECTRE, a MCDM algorithm, to the problem of VHO. This combination decreases the influence of the dissimilar, imprecise, and contradictory measurements for the VHO criteria coming from different sources. A performance analysis is done and the results are compared with traditional algorithms for $\mathrm{VHO}$. These results demonstrate a significant improvement with our developed algorithm.
\end{abstract}

\section{KEYWORDS}

Vertical Handover, Heterogeneous Networks, Fuzzy Logic, MCDM, ELECTRE

\section{INTRODUCTION}

In the next generation heterogeneous wireless networks, a user with a multi-interface terminal may have network access from different service providers using various technologies. Vertical Handover (VHO) is the capability to switch on-going connections from one Radio Access Network (RAN) to another. This switching is based on the discovered accesses, QoS constraints, operator policies, user preferences and available system capacity and utilization. Optimizing the VHO process is an important issue of research, which leads to reduction of network signaling and mobile device power loss and on the other hand improves network Quality of Service (QoS) and Grade of Service (GoS).

The authors in paper [1] propose multiple attribute decision making handover decision algorithm for WiMAX and Wi-Fi networks. The Analytic Hierarchy Process (AHP) is used to calculate the weights of various traffic parameters and the Simple Additive Weighting (SAW) and Multiplicative Exponent Weighting (MEW) processes are applied to calculate the QoS score function. The authors in [2] present a novel solution to the problem of vertical handoff for wireless networks when a mobile node carries multiple communication sessions with different DOI : $10.5121 /$ ijcnc.2011.3409 
International Journal of Computer Networks \& Communications (IJCNC) Vol.3, No.4, July 2011

QoS requirements. They extend the classical AHP method to provide the mobile node with two types of solutions: a deterministic solution and a probabilistic one. Paper [3] presents a cooperative vertical handover algorithm which considers not only the QoS factors but also the signalling cost and the load balance. The QoS information is gathered by the mobile terminal and the Grey Relational Coefficient (GRC) of the QoS part is calculated. Paper [4] proposes an optimal distributed network selection scheme in heterogeneous wireless networks considering multimedia application layer QoS. In this paper, an application layer QoS and the price of different networks are considered. Paper [5] proposes a novel speed sensitive vertical handoff algorithm based on fuzzy control. Based on collected information on RSS, load conditions of candidate networks, velocity of mobile terminals and user preference, the vertical handover decision is made by fuzzy logic process. Paper [6] considers the speed as an aspect of motion as input parameters for the handover decision. Two novel methods for speed estimation are proposed: Velocity Aware Handover Approach (VAHA) is the more simple approach and it uses a simple mathematical equation for estimation of the speed, and Angle of Arrival VAHA (AoA_VAHA) that is more complex and it uses the Angle of Arrival as additional information for the handover decision. In paper [7], the authors address the integration of IEEE802.11 WLANs and IEEE802.16 WMANs, focusing on the handover management aspects. However, the definition of trigger mechanism is not addressed in these approaches. In paper [8], a fuzzy decision making is described with consideration on a variety of context parameters such as RSS, Variation of the RSS (VRSS), bandwidth, traffic status and handover preference. The authors divide handovers into two parts: forced and voluntary handovers. In paper [9], the authors develop an adaptive variable threshold vertical handoff algorithm, which makes handoff decision after analyzing the signal strength fluctuation caused by slow fading through Fast Fourier Transform (FFT). This algorithm reduces handoff numbers and makes full use of WLAN resources. The authors in paper [10] propose a new vertical handover scheme for a VoIP connection, which makes use of the on-off characteristics of voice traffic by aligning the mutual silence period of the two parties engaged in conversation with the service disruption time as much as possible during the handover procedure. F. Jiadi et al. in [11] propose a useradaptive vertical handover scheme based on Media Independent Handover (MIH) function in the integration of Wi-Fi, WiMAX and UMTS networks environment. Users' QoS demands are given full consideration throughout the whole handover procedure. S. Rizvi et al. in paper [12] define a vertical handoff decision algorithm for real time services which provides a suitable mechanism for vertical handoff for the users roaming across heterogeneous wireless networks. A. Ahmed et al. in paper [13] propose an agent-based approach for vertical handover in heterogeneous wireless networks. They define a set of parameters that helps to decide, when and where to perform the handover by using the agent behaviors. Paper [14] tries to maintain the connectivity of a established call between two different networks using mobility management and proposes an efficient algorithm which maintain the real time connection as well as preventing the data loss during transition. Paper [15] proposes a new vertical handover scheme based on the prediction of mobility of mobile node. The proposed approach considers the movement velocity and direction of the MN, and Received Signal Strength (RSS) for accurate prediction of the MN's movement toward a specific access point. Authors of paper [16] provide a generic framework for handover decision management in next generation networks. They propose a handover decision algorithm that utilizes their generic framework and selects the network for handover such that the quality of experience of the user is near optimal. 
International Journal of Computer Networks \& Communications (IJCNC) Vol.3, No.4, July 2011

\section{ELECTRE AND FLC}

ELECTRE is a family of multi-criteria decision analysis methods that originated in Europe in the mid-1960s and it was developed by Bernard Roy [17][18]. The acronym ELECTRE stands for: ELimination Et Choix Traduisant la REalité (ELimination and Choice Expressing REality). ELECTRE has found vast applications in engineering decision making problems. The method performs pair-wise comparisons among alternatives for each one of the attributes separately to establish outranking relationships between the alternatives. The basic elements of this method is concordance and measures which are the set of all criteria for which alternative $i$ is not worse than the competing alternative $j$ and disconcordance measures which are the set of all criteria for which alternative $i$ is worse than the competing alternative $j$. These indicators are calculated for all pairs of alternatives and then the alternatives with the highest concordance value and with the lowest disconcordance value are found. There are formulas suggesting to determine overall score for each alternative based on these indicators.

Fuzzy Logic Control (FLC) is a non-linear control method, which attempts to apply the expert knowledge of an experienced user to the design of a controller. The fuzzy control system contains four main parts, the fuzzifier, the fuzzy rules base, the fuzzy inference engine, and the defuzzifier. The fuzzifier maps the real valued numbers into a fuzzy set, which is the input to the fuzzy inference engine. The fuzzifiction process includes the definition of the universe of discourse and the specification of the linguistic variables, the fuzzy sets for the linguistic variables, and the membership functions for the specified fuzzy sets. The fuzzy rules base consists of a collection of fuzzy IF-THEN rules to represent the human knowledge about the problem. The fuzzy inference engine maps the input fuzzy sets into output fuzzy sets and handles the way in which the rules are combined just as humans use many different types of inferential procedures. The defuzzifier task is the reverse operation to the fuzzifier. It maps the output fuzzy sets into real valued numbers.

\section{VHO SOLUTION}

In order to formulate the $\mathrm{VHO}$ as MCDM problem, Table 1 presents a representative set of VHO criteria that are considered in the decision making process using FL and ELECTRE.

Table 1: VHO criteria used in our VHO solution

\begin{tabular}{|l|l|l|}
\hline Attribute & Abbrev & Brief Explanation \\
\hline Resource Availability & RA & $\begin{array}{l}\text { to avoid any network congestion, the new or } \\
\text { handoff calls are usually connected to the network } \\
\text { with higher available resources. }\end{array}$ \\
\hline Received Signal Strength & RSS & $\begin{array}{l}\text { to connect the user to the network with the } \\
\text { strongest received signal and avoid unnecessary } \\
\text { handover, call drop, and packets or bits errors. }\end{array}$ \\
\hline Mobile Station Speed & MSS & $\begin{array}{l}\text { to avoid unnecessary handover overhead, the low } \\
\text { speed users are usually connected to the RAT with } \\
\text { small coverage area and the high speed users are } \\
\text { connected to the ANs with large coverage area. }\end{array}$ \\
\hline Service Types & ST & $\begin{array}{l}\text { due to the different QoS architectures used by the } \\
\text { different networks, some networks such as WLAN }\end{array}$ \\
\hline
\end{tabular}


International Journal of Computer Networks \& Communications (IJCNC) Vol.3, No.4, July 2011

\begin{tabular}{|l|l|l|}
\hline & & $\begin{array}{l}\text { are preferred for data, bursty services, and } \\
\text { streaming multimedia services and other networks } \\
\text { such as 3G networks are preferred for voice and } \\
\text { conversational multimedia services. }\end{array}$ \\
\hline User Preferred Price & UPP & $\begin{array}{l}\text { the operators assign the links of high cost networks } \\
\text { (with better QoS) for users who are willing to pay } \\
\text { more and the links of low cost networks to other } \\
\text { users. }\end{array}$ \\
\hline Security & SEC & $\begin{array}{l}\text { for some applications, confidentiality or integrity } \\
\text { of the transmitted data can be critical. For this } \\
\text { reason, a network with higher security level may } \\
\text { be chosen over another one which would provide } \\
\text { lower level of data security. }\end{array}$ \\
\hline
\end{tabular}

Our proposed solution has two main components. The first component is a set of small parallel FL subsystems that receive the different input criteria values from the different sources. Every FL subsystem give initial ranking value for the different alternatives according to one criterion. The second component is an MCDM system that takes the outputs of the FL subsystems as its input and it calculates the total ranking value for all alternatives. In the following two subsections, the parallel FL and MCDM components are described.

\subsection{The Parallel FL Component}

Our VHO solution contains six FL based subsystems. Each subsystem considers one of the VHO criteria mentioned in Table 1. The RA subsystem considers the resources availability criterion. The RSS subsystem considers the received signal strength criterion. The MSS subsystem considers the mobile station speed criterion. The ST subsystem considers the service type criterion. The UPP subsystem considers the user preference and price criterion. The SEC subsystem considers the security criterion. All the above subsystems have the following common characteristics.

- They use Mamdani style fuzzy inference system.

- They use the centroid method for defuzzification.

- Every subsystem has $x$ output variables, where $x$ is the number of existed RATs. Every output variable describes the probability of acceptance for the handoff request in one of the existed RATs.

- Each output variable has four membership functions \{TR (Totally Reject), PR (Probability Reject), PA (Probability Accept), and TA (Totally Accept)\}. Figure 1 shows a sample for an output variable with its membership functions. 


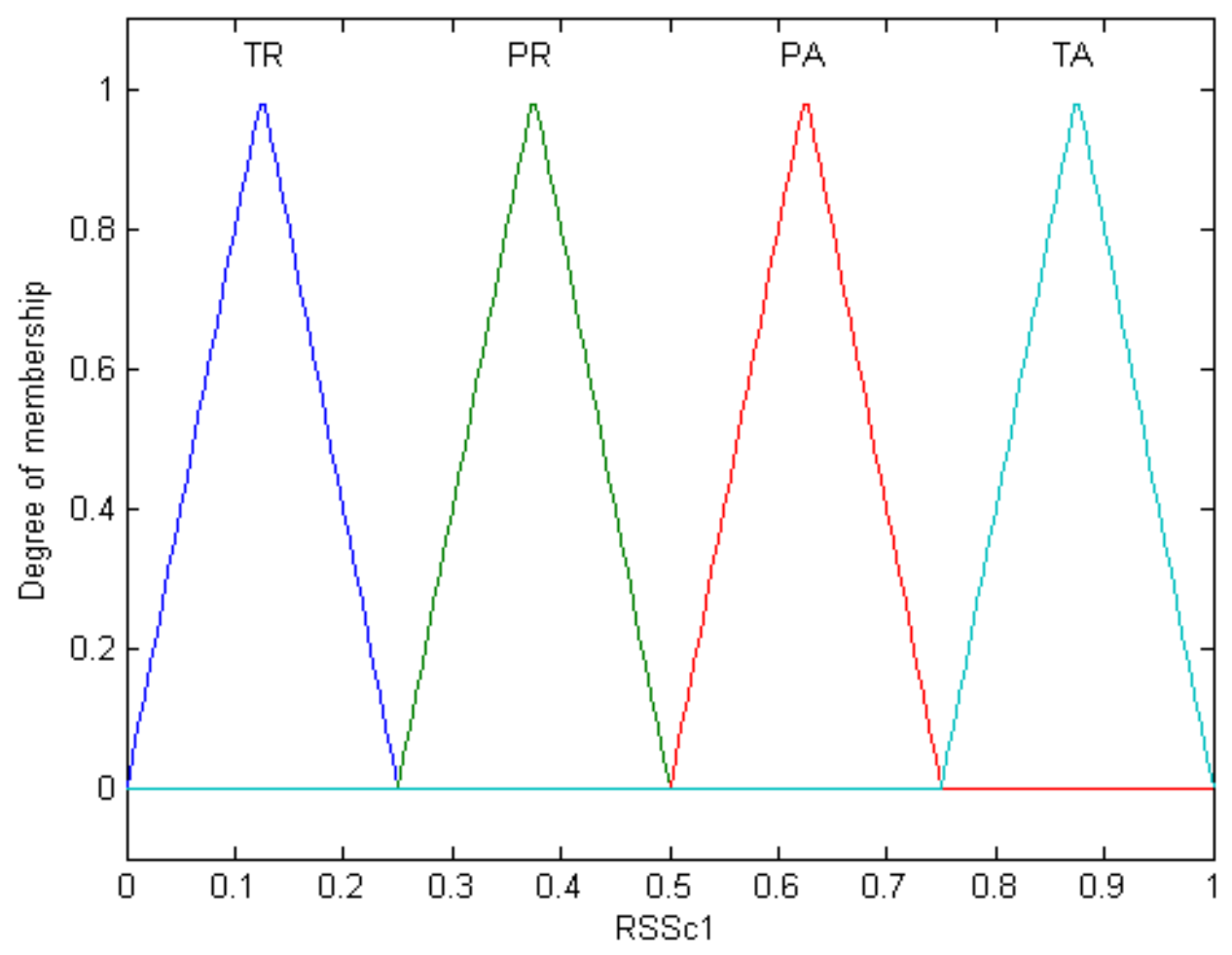

Figure 1: Output variable RSSc1

In addition to the above common characteristic, every subsystem has its own special characteristics. For example, the ST subsystem has two input variables, the first is "DelayReqc" to describe the one-way delay needed for the required service and the second is "RateReqc" to describe the bit rate needed for the required service. Both variables have three membership functions $\{$ High, Medium, Low $\}$. If we assume three existed RATs, then there will be three output variables " $S T_{c 1}, S T_{c 2}, S T_{c 3}$.". The Figures 2 and 3 show the membership functions of the "DelayReqc" and "RateReqc" input variables. The subsystem has also nine rules as shown in Table 2. 
International Journal of Computer Networks \& Communications (IJCNC) Vol.3, No.4, July 2011

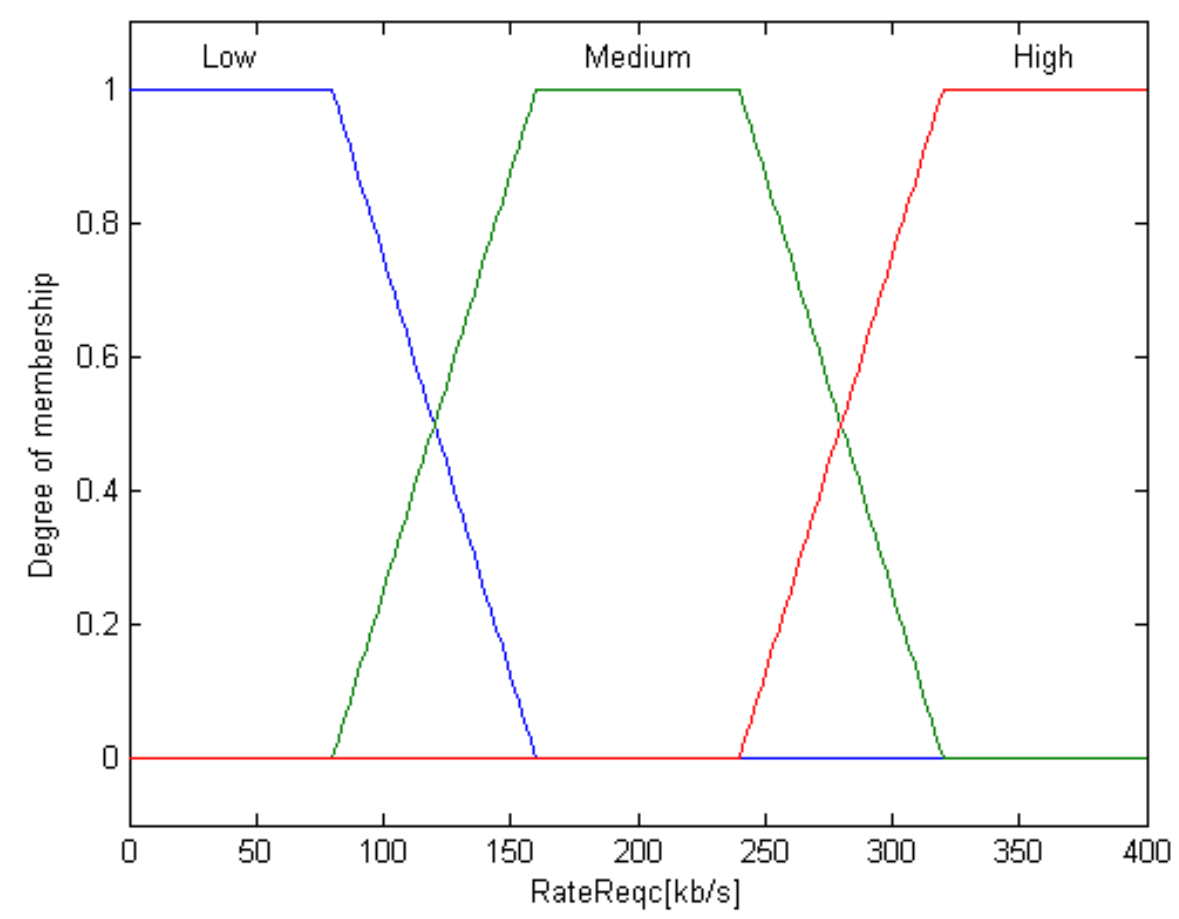

Figure 2: The input variable "DelayReqc"

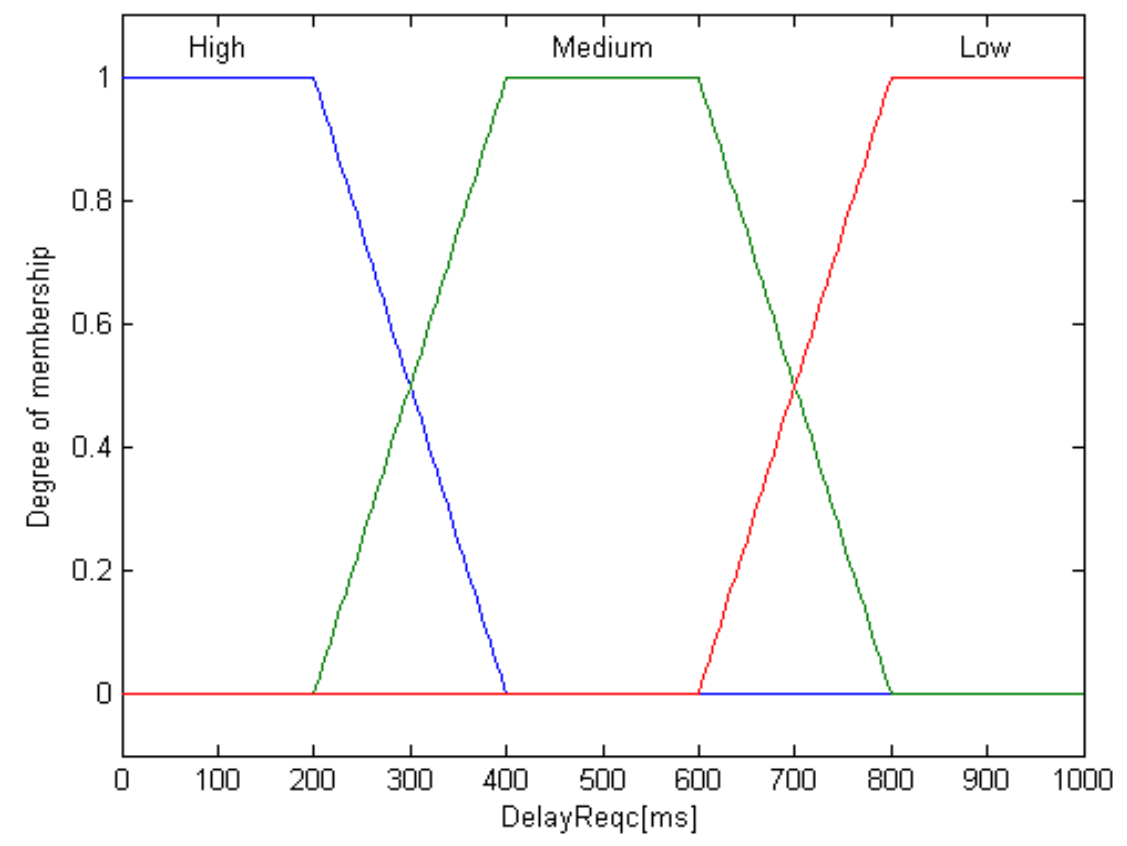

Figure 3: input variable "RateReqc" 
International Journal of Computer Networks \& Communications (IJCNC) Vol.3, No.4, July 2011

Table 2: The inference rules of the ST fuzzy based system

\begin{tabular}{|c|c|c|c|c|c|}
\hline Rule No. & DelayReqc & RateReqc & $S T_{c 1}$ & $S T_{c 2}$ & $S T_{c 3}$ \\
\hline 1 & $\mathrm{H}$ & $\mathrm{L}$ & $\mathrm{TA}$ & $\mathrm{TR}$ & PR \\
\hline 2 & $\mathrm{H}$ & $\mathrm{M}$ & PA & PR & PA \\
\hline 3 & $\mathrm{H}$ & $\mathrm{H}$ & PA & PA & PA \\
\hline 4 & $\mathrm{M}$ & $\mathrm{L}$ & PA & PR & PA \\
\hline 5 & $\mathrm{M}$ & $\mathrm{M}$ & PA & PA & PA \\
\hline 6 & $\mathrm{M}$ & $\mathrm{H}$ & PR & PA & PA \\
\hline 7 & $\mathrm{~L}$ & $\mathrm{~L}$ & PA & PA & PA \\
\hline 8 & $\mathrm{~L}$ & $\mathrm{M}$ & TR & TA & TA \\
\hline 9 & $\mathrm{~L}$ & H & TR & TA & PA \\
\hline
\end{tabular}

\subsection{The MCDM Component}

The input criteria of the MCDM are the outputs of the FL based control subsystems. Since all the outputs of FL subsystems are in the range $[0,1]$, there is not any need to scale the criteria performance against alternatives. The decision matrix for the different alternatives against the set of criteria can be written as shown in equation 1 .

$$
N W=\left[\begin{array}{cccccc}
R A_{1} & R S S_{1} & M S S_{1} & S T_{1} & U P P_{1} & S E C_{1} \\
R A_{2} & R S S_{2} & M S S_{2} & S T_{2} & U P P_{2} & S E C_{2} \\
\vdots & \vdots & \vdots & \vdots & \vdots & \vdots \\
R A_{N} & R S S_{N} & M S S_{N} & S T_{N} & U P P_{3} & S E C_{N}
\end{array}\right]
$$

The value of each of the attributes in the decision matrix is compared with a corresponding reference attribute value as shown in equation (2).

$$
N W_{\text {ref }}=\left[\begin{array}{llllll}
R A_{\text {ref }} & R S S_{\text {ref }} & M S S_{\text {ref }} & S T_{\text {ref }} & U P P_{\text {ref }} & S E C_{\text {ref }}
\end{array}\right]
$$

An absolute difference between the two values is taken to calculate a new matrix called $\boldsymbol{N} \boldsymbol{W}_{\boldsymbol{A d j}}$ as shown in equation 3 .

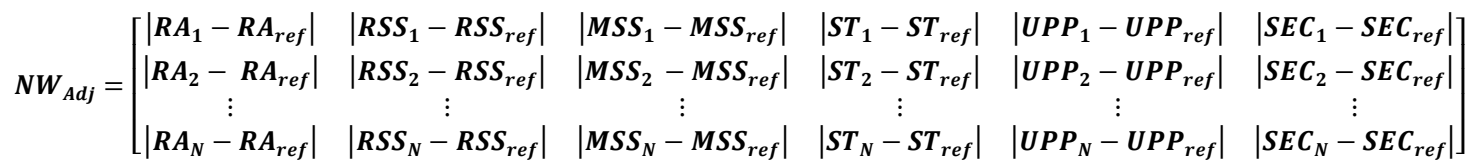

The $\boldsymbol{N} \boldsymbol{W}_{\boldsymbol{A} \boldsymbol{d} \boldsymbol{j}}$ matrix can be rewritten in more easier way as shown in equation (4).

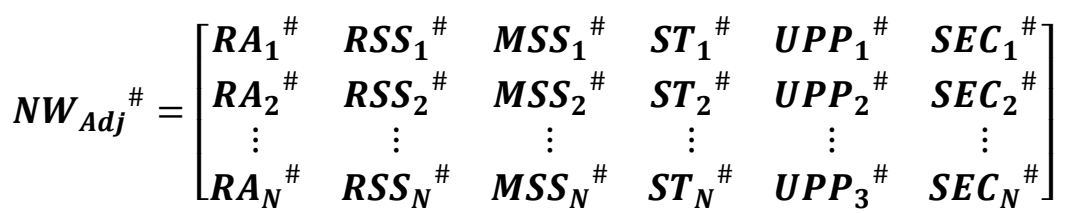


International Journal of Computer Networks \& Communications (IJCNC) Vol.3, No.4, July 2011

Now, is necessary take into consideration the relative importance of each of the attributes involved in the decision about network selection. For the $j$-th attribute is assigned a weight $w$ j. $W$ is the total weight and is calculated using (5).

$$
W=W_{R A}+W_{R S S}+W_{M S S}+W_{S T}+W_{U P P}+W_{S E C}=1
$$

Using these assigned weights, the matrix in equation (4) is updated as shown in (6).

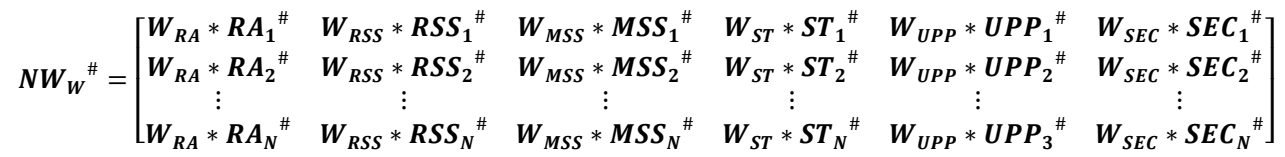

Pair-wise comparisons of networks is calculated to obtain the concordance set $\operatorname{CSet}(k, l)$ indicating the attribute of network $k$ is better than network $l$ and the discordance set DSet $k$, $\zeta)$ indicating the attribute of network $k$ is worse than network $l$. The concordance and discordance sets for the first two alternatives are formed as shown in equations 7 and 8.

$$
\begin{aligned}
& \operatorname{CSet}_{12}=\left\{j:\left(N W_{W}^{\#}\right)_{1, j} \geq\left(N W_{W}^{\#}\right)_{2, j}\right\}(7) \\
& \operatorname{DSet}_{12}=\left\{j:\left(N W_{W}^{\#}\right)_{1, j}<\left(N W_{W}^{\#}\right)_{2, j}\right\}(8)
\end{aligned}
$$

For $N$ alternatives, the concordance matrix $\mathbf{C}$ can be represented as shown in (9).

$$
C=\left[\begin{array}{ccccc}
- & C_{12} & C_{13} & \cdot & C_{1 N} \\
C_{21} & - & C_{23} & \cdot & C_{2 N} \\
C_{31} & C_{32} & - & \cdot & C_{3 N} \\
\cdot & \cdot & \cdot & \cdot & \cdot \\
C_{N 1} & C_{N 2} & C_{N 3} & \cdot & -
\end{array}\right]
$$

The items of the concordance matrix $\mathrm{C}$ are calculated as shown in (10).

$$
\mathrm{C}_{\mathrm{kl}}=\sum_{J \in C S e t} W_{j l}
$$

In a similar way, the discordance matrix D can be represented as shown in (11).

$$
D=\left[\begin{array}{ccccc}
- & D_{12} & D_{13} & \cdot & D_{1 N} \\
D_{21} & - & D_{23} & \cdot & D_{2 N} \\
D_{31} & D_{32} & - & \cdot & D_{3 N} \\
\cdot & \cdot & \cdot & \cdot & \cdot \\
D_{N 1} & D_{N 2} & D_{N 3} & \cdot & -
\end{array}\right]
$$

The items of the discordance matrix D are calculated as shown in (12).

$$
\mathbf{d}_{\mathrm{kl}}=\frac{\sum_{J \in D S e t_{k l}}\left|\left(N W_{W}^{\#}\right)_{k j}-\left(N W_{W}^{\#}\right)_{l j}\right|}{\sum_{j}\left|\left(N W_{W}^{\#}\right)_{k j}-\left(N W_{W}^{\#}\right)_{l j}\right|}
$$


International Journal of Computer Networks \& Communications (IJCNC) Vol.3, No.4, July 2011

The next step is to determine the concordance and discordance dominance matrices. The concordance dominance matrix is calculated using a threshold value for the concordance index. A way to determine threshold value, Cthreshold, is to use the average concordance index as shown in equation (13).

$$
C_{\text {threshold }}=\frac{\sum_{k=1}^{N} \sum_{l=1}^{N} c_{k l}}{N *(N-1)}
$$

The elements of concordance dominance matrix, $\boldsymbol{C d o m}$, are calculated using the Cthreshold value as shown in equation (14).

$$
\left(C_{d o m}\right)_{k l}=1: c_{k l} \geq C_{\text {threshold }} \quad \& \quad\left(C_{d o m}\right)_{k l}=0: c_{k l}<C_{\text {threshold }}
$$

The discordance dominance matrix is calculated using a similar threshold value, Dthreshold. This value can be calculated using a similar formula as shown in equation (15).

$$
D_{\text {threshold }}=\frac{\sum_{k=1}^{N} \sum_{l=1}^{N} d_{k l}}{N *(N-1)}
$$

The elements of the discordance dominance matrix, Ddom, are calculated using the Dthreshold value as shown in equation (16).

$$
\left(D_{\text {dom }}\right)_{k l}=1: d_{k l} \geq C_{\text {threshold }} \quad \& \quad\left(D_{\text {dom }}\right)_{k l}=0: d_{k l}<C_{\text {threshold }}
$$

The aggregate dominance matrix, $\boldsymbol{A d o m}$, is calculated as shown in equation (17).

$$
\left(\boldsymbol{A}_{\text {dom }}\right)_{k l}=\left(\boldsymbol{C}_{\text {dom }}\right)_{k l} *\left(D_{d o m}\right)_{k l}(17)
$$

The aggregate dominance matrix, Adom, only provides partial preference ordering of the access networks under consideration and it does not provide a complete ranking for all the alternatives. To give full ranking for the alternatives two new parameters, $C i$ and $D i$, are used.

Parameter $C_{i}$ is called the net concordance index and it is a measure of dominance of an alternative $i$ over other alternatives when compared with a measure of dominance of other alternatives over the alternative. It can be calculated as shown in equation (18).

$$
C_{i}=\sum_{j=1}^{N} C_{i j}-\sum_{j=1}^{N} C_{j i} \text { (18) }
$$

In a similar way, the term net discordance index $D_{i}$, is defined as a measure of relative weakness of alternative $i$ over other alternatives when compared with a measure of weakness of other alternatives from the alternative $i$.

$$
D_{i}=\sum_{j=1}^{N} D_{i j}-\sum_{j=1}^{N} D_{j i} \text { (19) }
$$

An alternative with the highest value of net concordance index $C$ and lowest value of net discordance index $D$ would be preferred. 
International Journal of Computer Networks \& Communications (IJCNC) Vol.3, No.4, July 2011

\section{THE PERFORMANCE EVALUATION}

Our proposed solution is evaluated using the simulation approach. This section presents the used performance metrics and simulation models.

\subsection{The Performance Metrics}

In this paper four performance evaluation metrics have been used to evaluate our algorithm against several reference algorithms. The used metrics can be described briefly as follows.

Handover rate $(\mathbf{P n})$ : reducing the number of handovers is usually preferred as frequent handovers would cause wastage of network resources. Pn can be calculated as shown in equation 20.

$$
P_{\mathbf{n}}=\frac{\text { Number of Handoff Requests }}{\text { Total Number of Users }}
$$

Handover failure rate (Pf): a handover failure occurs when the handover is initiated but the target network does not have sufficient resources to complete it, or when the mobile terminal moves out of the coverage of the target network before the process is analyzed. Pf can be calculated as shown in equation (21).

$$
P_{\mathbf{f}}=\frac{\text { Number of Unsuccessful Handoff of Requests }}{\text { Number of Handoff Requests }}
$$

The percentage of users who are assigned to networks of their preference $(\mathrm{Pu})$ : this metric reflects the user point of view about the performance of the selection process.

The usage percentage of the low cost network resources $\left(\mathbf{P}_{0}\right)$ : this metric reflect the operator point of view because it utilizes the resources of the high cost networks (i.e., WMAN and WWAN). Simply, Po can be calculated as the percentage between the number of users in WLAN and the total number of users as shown in equation (22).

$$
P_{0}=\frac{\text { Number of users in WLAN }}{\text { Total Number of Users }}
$$

\subsection{The Simulation Environment}

A modified version of MATLAB based simulator called RUNE [19], [20] has been used. The simulation environment defines a system model, a mobility model, a propagation model, and services model. The system model considers the coexistence of three types of wireless access networks.

The first network is a CDMA based WWAN with seven macro cells and cell radius of 900m. The second one is a CDMA based WMAN with twelve macro cells and cell radius of $3500 \mathrm{~m}$. The third one is a CDMA based WLAN with eighty four micro cells and cell radius of $80 \mathrm{~m}$. All cells have standard hexagonal shapes with Omni-directional antennas. 
International Journal of Computer Networks \& Communications (IJCNC) Vol.3, No.4, July 2011

The mobiles are randomly distributed over the system. In every slot each mobile is moved a random distance in a random direction at defined time steps. The movement pattern of each mobile depends on the velocity and acceleration. The velocity is a vector quantity with magnitude and direction. The velocity of the ith mobile is updated according to equation (23).

$$
\mathbf{V}_{\mathbf{i}}=\mathbf{V}_{\mathrm{I}-\mathbf{1}} \cdot \mathbf{P}+\sqrt{\mathbf{1 - \mathbf { P } ^ { 2 }}} \cdot \mathbf{V}_{\mathrm{m}} \cdot \mathbf{X}
$$

where $\mathrm{Vi}$ is the complex speed [m/s]. Vi-1 is the complex speed in the previous time step. $\mathrm{X}$ is a Rayleigh distributed magnitude with mean 1 and a random direction. $\mathrm{Vm}$ is the mean speed of mobiles. $\mathrm{P}$ is the correlation of the velocity between time steps. $\mathrm{P}$ depends on both amean which is the mean acceleration of the mobile user and Vmean. Vm has been set to $5[\mathrm{~m} / \mathrm{s}]$ and the mean acceleration has been set to 1 .

The propagation model simulates the different losses and gains during the signal propagation between the transmitter and the receiver in the system environment. The wireless propagation model used in this paper is described in a logarithmic scale as in equation (24).

$$
G=G_{D}+G_{F}+G_{R}+G_{A}
$$

Equation 24 contains four components; the first component is the distance attenuation GD that is calculated by Okumura- Hata formula presented in [21]. The second component is the shadow fading GF that is modeled as a log-normal distribution with standard deviation of $6 \mathrm{~dB}$ and $0 \mathrm{~dB}$ mean. The third component is the Rayleigh fading GR that is modeled using a Rayleigh distribution. The forth component is the antenna gain GA that adds the antenna gain in $\mathrm{dB}$.

Adaptive service model is considered in our simulation. The service $i$ is mainly characterized by its bit rate requirement "RateReqc" and delay requirement "DelayReqc". The users are generated according to Poisson process. The service holding time is exponential distribution with mean holding time equals to 120 seconds.

\section{THE RESULTS STUDY}

Three different reference algorithms are simulated and evaluated against our developed solution. The first algorithm is a terminal speed based VHO where high speed users are sent to the high-coverage network and the low and medium speed users are sent to the smaller coverage area networks. The second algorithm is a resource availability based VHO where the users are assigned to the network with higher resources. The third algorithm is based on a received signal VHO where the users are assigned to the network with higher signal strength. Some simulation results for different sets of users are presented in this section.

From both Figure 4 and the numerical samples for $P_{n}$ values shown in Table 3, the reduction in the number of handovers in our solution can be seen. For example, with 1366 users in the environment, the handover rate with the terminal-speed based algorithm is $23.3 \%, 29.2 \%$ with 
International Journal of Computer Networks \& Communications (IJCNC) Vol.3, No.4, July 2011

the resource availability based algorithm, and $27.2 \%$ with the signal strength based algorithm. The same number with the combined FL and ELECTRE algorithm is around 19.5\%. In general, our developed FL- ELECTRE solution achieves around 3\% enhancement over the terminal speed based algorithm and around 7\% over the resource availability and the signal strength based algorithms.

Table 3: Pn values of the combined FL and ELECTRE based algorithm against the reference algorithms

\begin{tabular}{ccccc}
\hline $\begin{array}{c}\text { No. of } \\
\text { Users }\end{array}$ & $\begin{array}{c}\text { FL-ELECTRE based } \\
\text { VHO Pn }\end{array}$ & $\begin{array}{c}\text { Terminal Speed } \\
\text { based VHO Pn }\end{array}$ & $\begin{array}{c}\text { Resource availability } \\
\text { based VHO Pn }\end{array}$ & $\begin{array}{c}\text { Signal Strength } \\
\text { based VHO Pn }\end{array}$ \\
\hline 580 & 0.224 & 0.239 & 0.247 & 0.274 \\
702 & 0.253 & 0.227 & 0.276 & 0.295 \\
857 & 0.220 & 0.239 & 0.305 & 0.300 \\
976 & 0.204 & 0.260 & 0.298 & 0.280 \\
1086 & 0.184 & 0.242 & 0.274 & 0.292 \\
1180 & 0.225 & 0.236 & 0.266 & 0.284 \\
1276 & 0.191 & 0.262 & 0.291 & 0.274 \\
1366 & 0.195 & 0.233 & 0.292 & 0.272 \\
\hline
\end{tabular}

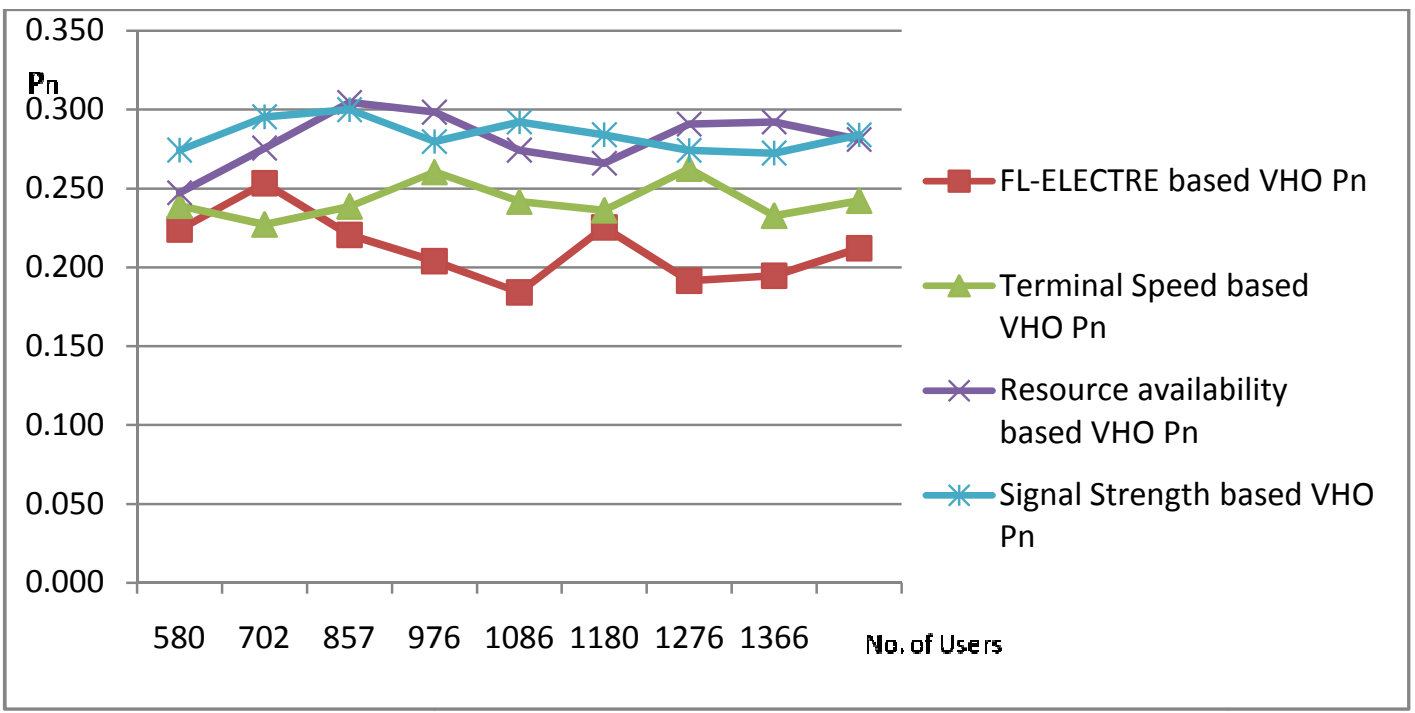

Figure 4: Pn values of the combined FL and ELECTRE based algorithm against the reference algorithms

From both Figure 5 and the numerical samples for $\mathrm{Pf}_{\mathrm{f}}$ values shown in Table 4, the enhancement in the handover failure rate in our solution can be seen. For example, with 1366 users in the environment, the handover failure rate with the terminal-speed based algorithm is $25.4 \%$, $26.5 \%$ with the resource availability based algorithm, and $18.3 \%$ with the signal strength based algorithm. The same number with the combined FL and ELECTRE algorithm is around 18\%. 
International Journal of Computer Networks \& Communications (IJCNC) Vol.3, No.4, July 2011

In general, our developed FL-ELECTRE solution achieves around 8\% enhancement over the terminal speed based algorithm and around $4 \%$ over the resource availability based algorithm. Our algorithm achieve a comparable results with the signal strength based algorithm.

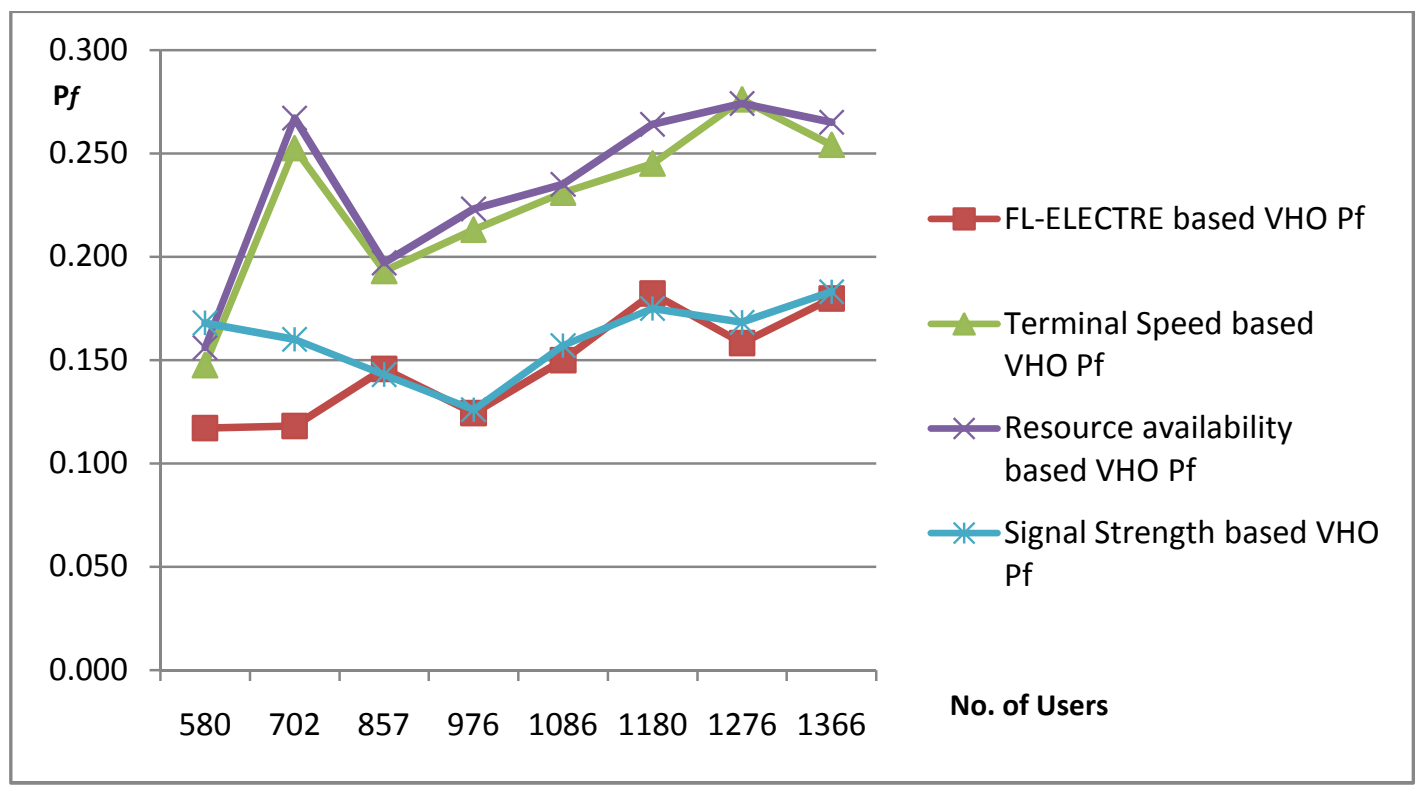

Figure 5: Pf values of the combined FL and ELECTRE based algorithm against the reference algorithms

Table 4: Pf values of the combined FL and ELECTRE based algorithm against the reference algorithms

\begin{tabular}{ccccc}
\hline $\begin{array}{c}\text { No. of } \\
\text { Users }\end{array}$ & $\begin{array}{c}\text { FL-ELECTRE } \\
\text { based VHO } \boldsymbol{P}_{\boldsymbol{f}}\end{array}$ & $\begin{array}{c}\text { Terminal Speed } \\
\text { based VHO } \boldsymbol{P}_{f}\end{array}$ & $\begin{array}{c}\text { Resource availability } \\
\text { based VHO } \boldsymbol{P}_{f}\end{array}$ & $\begin{array}{c}\text { Signal Strength } \\
\text { based VHO } \boldsymbol{P}_{\boldsymbol{f}}\end{array}$ \\
\hline 580 & 0.117 & 0.147 & 0.156 & 0.168 \\
702 & 0.118 & 0.252 & 0.267 & 0.160 \\
857 & 0.146 & 0.193 & 0.197 & 0.143 \\
976 & 0.124 & 0.213 & 0.223 & 0.126 \\
1086 & 0.150 & 0.231 & 0.235 & 0.157 \\
1180 & 0.182 & 0.245 & 0.264 & 0.175 \\
1276 & 0.158 & 0.276 & 0.274 & 0.168 \\
1366 & 0.180 & 0.254 & 0.265 & 0.183 \\
\hline
\end{tabular}

From both Figure 6 and the numerical samples for Po values shown in Table 5, the great improvement in the percentage of the users who are assigned to low cost networks (i.e. WLAN) in our solution can be seen. For example, with 1366 users in the environment, the percentage of satisfied users with the terminal-speed based algorithm is $36.4 \%, 28.5 \%$ with the resource availability based algorithm, and $35.4 \%$ with the signal strength based algorithm. The same number with the combined FL and ELECTRE algorithm is around 40.6\%. In general, our developed FL-ELECTRE solution achieves around 4\% enhancement over the terminal speed 
International Journal of Computer Networks \& Communications (IJCNC) Vol.3, No.4, July 2011

based algorithm and around $13 \%$ and $8 \%$ over the resource availability based algorithm and the signal strength based algorithm respectively.

Table 5: Po values of the combined FL and ELECTRE based algorithm against the reference algorithms

\begin{tabular}{ccccc}
\hline $\begin{array}{c}\text { No. of } \\
\text { Users }\end{array}$ & $\begin{array}{c}\text { FL-ELECTRE } \\
\text { based VHO } \boldsymbol{P}_{\boldsymbol{o}}\end{array}$ & $\begin{array}{c}\text { Terminal Speed } \\
\text { based VHO } \boldsymbol{P}_{\boldsymbol{o}}\end{array}$ & $\begin{array}{c}\text { Resource availability } \\
\text { based VHO } \boldsymbol{P}_{\boldsymbol{o}}\end{array}$ & $\begin{array}{c}\text { Signal Strength } \\
\text { based VHO } \boldsymbol{P}_{\boldsymbol{o}}\end{array}$ \\
\hline 580 & 0.417 & 0.387 & 0.253 & 0.287 \\
702 & 0.450 & 0.364 & 0.287 & 0.342 \\
857 & 0.424 & 0.376 & 0.257 & 0.320 \\
976 & 0.415 & 0.397 & 0.297 & 0.365 \\
1086 & 0.415 & 0.364 & 0.276 & 0.354 \\
1180 & 0.403 & 0.397 & 0.286 & 0.343 \\
1276 & 0.419 & 0.364 & 0.275 & 0.375 \\
1366 & 0.406 & 0.364 & 0.285 & 0.354 \\
\hline
\end{tabular}

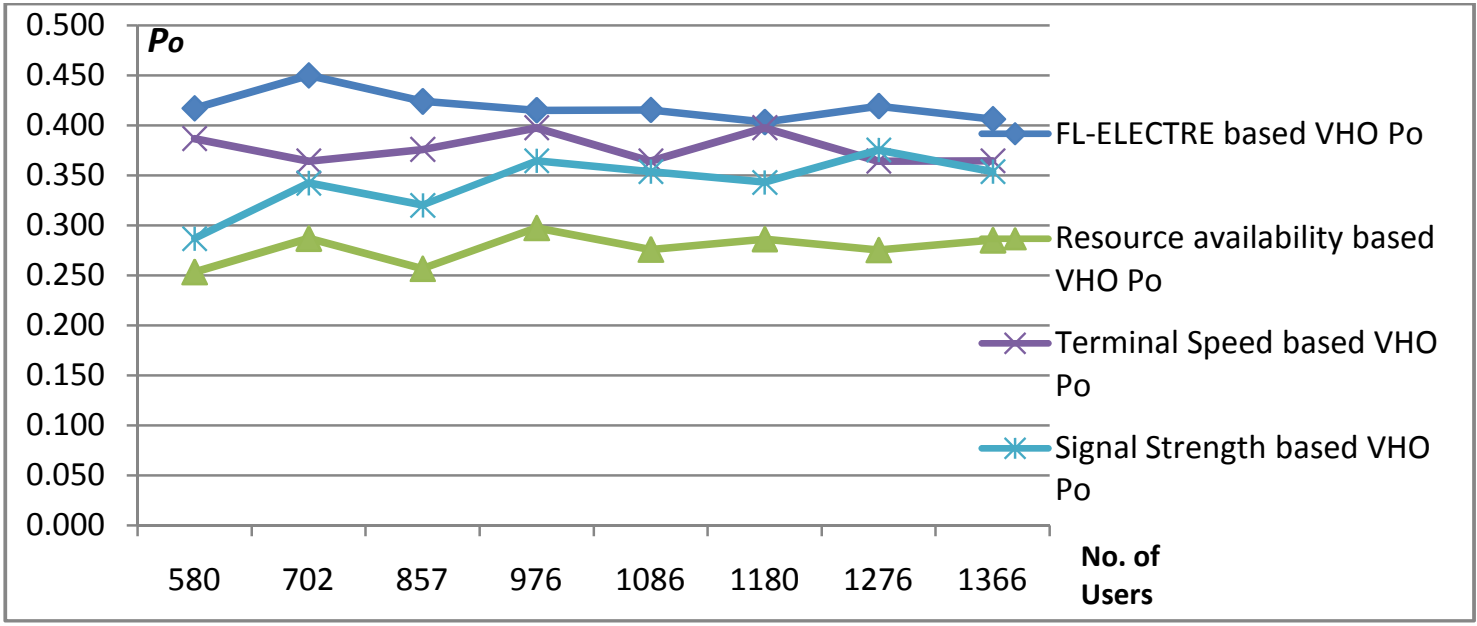

Figure 6: Po values of the combined FL and ELECTRE based algorithm against the reference algorithms

From both Figure 7 and the numerical samples for $P u$ values shown in Table 6, the great improvement in the percentage of the users who are assigned to the network of their preference in our solution can be seen. For example, with 1366 users in the environment, the percentage of satisfied users with the terminal-speed based algorithm is $34.8 \%, 38.5 \%$ with the resource availability based algorithm, and $35.3 \%$ with the signal strength based algorithm. The same number with the combined FL and ELECTRE algorithm is around 52.5\%. In general, our developed FL-ELECTRE solution achieves around 15\% enhancement over the terminal speed based algorithm and around $14 \%$ and $15 \%$ over the resource availability based algorithm and the signal strength based algorithm respectively. 
International Journal of Computer Networks \& Communications (IJCNC) Vol.3, No.4, July 2011

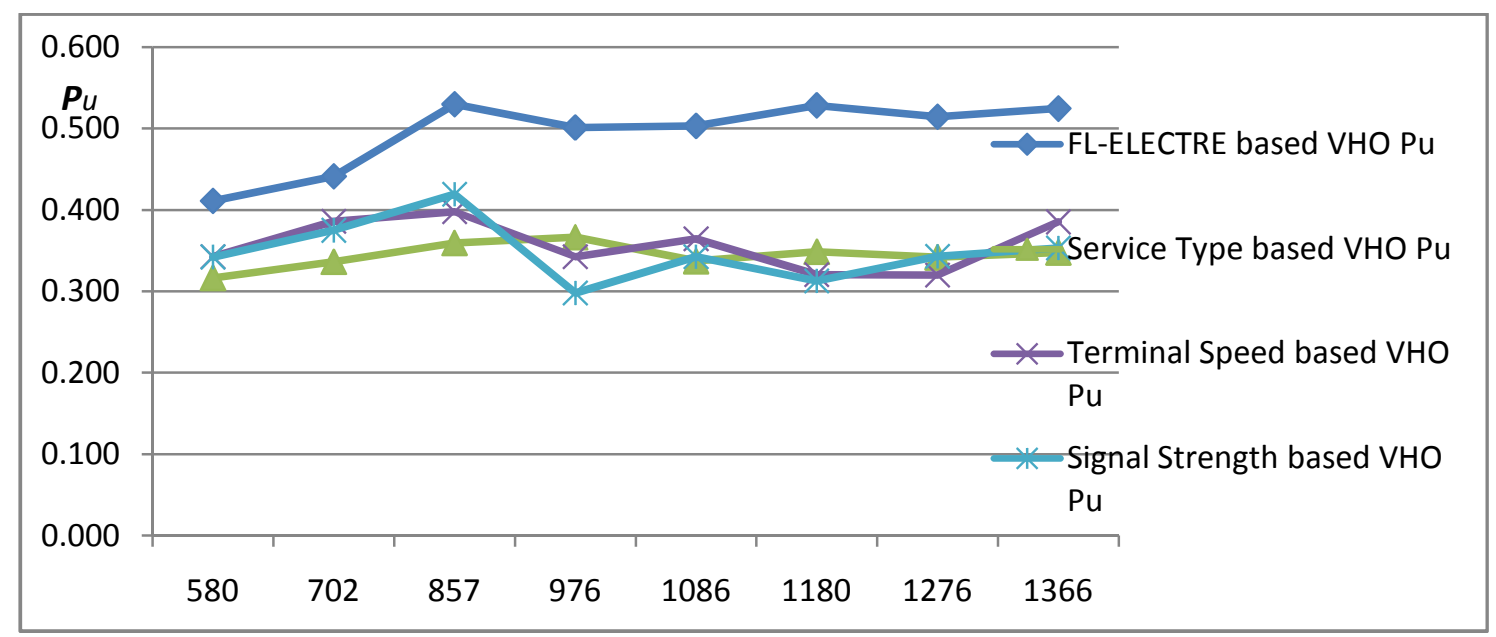

Figure 7: Pu values of the combined FL and ELECTRE based algorithm against the reference algorithms

Table 6: Pu values of the combined FL and ELECTRE based algorithm against the reference algorithms

\begin{tabular}{ccccc}
\hline $\begin{array}{c}\text { No. of } \\
\text { Users }\end{array}$ & $\begin{array}{c}\text { FL-ELECTRE } \\
\text { based VHO } \boldsymbol{P} u\end{array}$ & $\begin{array}{c}\text { Service Type } \\
\text { based VHO } \boldsymbol{P} u\end{array}$ & $\begin{array}{c}\text { Terminal Speed } \\
\text { based VHO } \boldsymbol{P} u\end{array}$ & $\begin{array}{c}\text { Signal Strength } \\
\text { based VHO } \boldsymbol{P} u\end{array}$ \\
\hline 580 & 0.411 & 0.317 & 0.342 & 0.342 \\
702 & 0.441 & 0.336 & 0.386 & 0.375 \\
857 & 0.530 & 0.359 & 0.398 & 0.419 \\
976 & 0.501 & 0.366 & 0.342 & 0.298 \\
1086 & 0.503 & 0.337 & 0.364 & 0.342 \\
1180 & 0.529 & 0.349 & 0.320 & 0.313 \\
1276 & 0.515 & 0.342 & 0.320 & 0.343 \\
1366 & 0.525 & 0.348 & 0.385 & 0.353 \\
\hline
\end{tabular}

\section{CONCLUSIONS AND FUTURE WORK}

The paper develops a novel operator algorithm for the VHO in co-existed WWAN, WMAN, and WLAN environment. The developed algorithm is based on the parallel FL decision and on the ELECTRE multiple criteria decision making tool. The developed solution is evaluated using simulation approaches. Its performance is compared against several reference algorithms. The simulation results show that the developed solution has a better and robust performance over the reference algorithm in terms of the handover rate number of successful handover, the operator benefits and the QoS. 
International Journal of Computer Networks \& Communications (IJCNC) Vol.3, No.4, July 2011

The developed algorithm has reduced the complexity involved in the heterogonous environment using the idea of small fuzzy based control subsystems with small sets of inference rules. The developed algorithm is scalable and is able to handle any number of RATs with a large set of criteria. The algorithm can cope with the different and contrast view points and goals of the operator and users and it can react to the accumulated human knowledge about the problem. The proposed algorithm has been used to present and design a multi criteria ANS solution that considered the user, the QoS, and the operator view points.

Different aspects of our work have to be developed. An optimum values for the weights of the different criteria can be found using a global searching method such as genetic algorithm. The rules and membership functions of the fuzzy subsystems can be built or tuned using the genetic algorithms or the neural networks. Our algorithm can be compared with more reference algorithms. Also, more performance metrics can be used to evaluate the different algorithms.

\section{REFERENCES}

[1] S.-F. Yang, J.-S. Wu, and H. Hsu-Hung, (2008) "A vertical media independent handover decision algorithm across Wi-Fi and WiMAX networks", 5th IFIP International Conference on Wireless and Optical Communications Networks (WOCN), pp. 1-5.

[2] R.A.V. Ramirez, and V.M. Ramos R, (2009) "A vertical handoff decision algorithm which considers the uncertainty during the decision making process", IFIP International Conference on Wireless and Optical Communications Networks (WOCN '09), pp 7-13.

[3] Shan Yang, Zhangdui Zhong, Rongtao Xu, (2009) "A cooperative vertical handover algorithm in integrated wireless and mobile network", 5th International Conference on Wireless Communications, Networking and Mobile Computing (WiCom '09), pp 1-7.

[4] Si Pengbo, F.R Yu, Ji Hong; V.C.M. Leung, (2009) "Optimal network selection in heterogeneous wireless multimedia networks", IEEE International Conference on Communications (ICC '09), pp 1-5.

[5] GE Kun, JI Hong, LI Xi, (2009) "A Speed sensitive vertical handoff algorithm based on fuzzy control", The 5th International Conference on Wireless communications, networking and mobile computing (WiCOM'09), pp 16-22.

[6] V. Rakovic, V. Atanasovski, L. Gavrilovska, (2009) "Velocity aware vertical handovers", 2nd International Symposium on Applied Sciences in Biomedical and Communication Technologies (ISABEL 2009), pp 1 - 6.

[7] A. Pontes, D. dos Passos Silva, J. Jailton, O. Rodrigues, K.L Dias, (2008)“Handover management in integrated WLAN and mobile WiMAX networks", IEEE Communication Magazine, pp. 86-95 
International Journal of Computer Networks \& Communications (IJCNC) Vol.3, No.4, July 2011

[8] A. Ezzouhairi, A. Quintero, S. Pierre, (2008 ) "A fuzzy decision making strategy for vertical handoffs," IEEE Canadian Conference on Electrical and Computer Engineering, Proceedings (CCECE 2008), pp 583-587

[9] X. Shengdong, W. Meng, (2008) "Adaptive variable threshold vertical handoff algorithm", IEEE International Conference Neural Networks and Signal Processing, ICNNSP, pp 366-369

[10] H.-H. Choi, O. Lee Song, (2009) "Voice-activity-based vertical handover in 3G-WLAN interworking networks", IEEE Electronics Letters , pp 1109 - 1101

[11] F. Jiadi, J. Hong, L. Xi, (2009) "User-adaptive vertical handover scheme based on MIH for heterogeneous wireless networks", 5th IEEE International Conference on Wireless Communications, Networking and Mobile Computing (WiCom09), pp 1 - 4

[12] S. Rizvi, A. Aziz, N.M. Saad, (2010) "Optimizations in vertical handoff decision algorithms for real time services", International Conference on Intelligent and Advanced Systems (ICIAS2010), pp 1 - 4

[13] A. Ahmed, R. Amoud, L. Merghem-Boulahia, D. Gaiti, (2010) "An agent-based approach for vertical handover in heterogeneous wireless networks", IEEE/ACS International Conference on Computer Systems and Applications (AICCSA2010), pp 1 - 8

[14] K. Javed, U. Saleem, K. Hussain, M. Sher, (2010) "An efficient approach for vertical handover between WLAN and EVDO", 2nd International Conference on Information Technology Convergence and Services (ITCS), pp 1 - 5

[15] B. Yle Jung, M. Seok Choi, H. Yong Youn, O. Song, (2010) "Vertical Handover based on the Prediction of Mobility of Mobile Node", 8th IEEE International Conference on Pervasive Computing and Communications Workshops (PERCOM Workshops), pp 534 - 539

[16] S. Menezes and S. Venkatesan, (2011) "A generic handover decision management framework for next generation networks", International Journal of Computer Networks \& Communications (IJCNC) Vol.3, No.2

[17] C.L. Hwang, K. Yoon, (1995) "Multiple Attribute Decision Making: An Introduction", Sage Publications

[18] M. Rogers, M. Bruen and L.-Y. Maystre, (2000) ELECTRE and Decision Support Methods and Applications in Engineering and Infrastructure Investment, Kluwer Academic Publishers.

[19] J. Zander and S. Kim, (2001) Radio Resource Management for Wireless Networks, Artech House

[20] P. Bruin, (1999) RUNE 5: Report T/B 99:036, Ericsson Radio Systems AB

[21] M. Hata, (1980) "Empirical formula for propagation loss in land mobile radio service", IEEE Transaction on Vehicular Technology, pp. 317-325 
International Journal of Computer Networks \& Communications (IJCNC) Vol.3, No.4, July 2011

\section{Authors}

Mohammed M. Alkhawlani is a Senior Lecturer at the University of Science and Technology (UST), Sana'a, Yemen. He received his PhD in Data Communication and Networking Engineering from De Montfort University, UK, in July 2008. He received his MSc in Data Communication Systems from Brunel University, UK, in December 2003. He received his BSc in Computer Engineering from Cairo University, Egypt, in July 2001.

His research interest is radio resource management in the next generation of wireless networks with the aid of artificial intelligence tools.

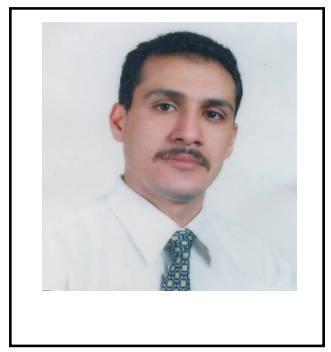

